\title{
ÉTUDE PONDÉRALE DU DÉVELOPPEMENT DES SOURIS ALOPÉCIQUES
}

\author{
N. KовоzIEFF. - Laboratoire de Génétique, École nationale vétérinaire, 94-Alfort.
}

Cette étude s'insère dans le travail d'ensemble dont le but est la mise en évidence des principales caractéristiques biométriques des diverses lignées normales ou stocks mutants en notre possession. Les premiers résultats concernent le développement pondéral des souris alopéciques. La forme d'alopécie décrite par nous est une anomalie de la peau, récessive, non liée au sexe, symbole "alp ». Flle est reconnaissable à la chute progressive des poils, suivie d'un épaississement de la peau, de formation de plis et d'hyperkeratose. Les souris étudiées ont un aspect normal pendant les 2 premières semaines après la naissance. La chute des poils débute aux orbites et s'étend toujours d'avant en arrière tout en respectant relativement longtemps les oreilles. La souris apparait "alopécique "(complètement nue) vers le $30^{\circ}$ jour. Les souris atteintes ont un bon état général jusqu'au début de l'épilation, puis, pendant toute la durée de la chute des poils, elles accusent un réel état de souffrance qui se caractérise par un retard de croissance par rapport aux sujets normaux de la même portée et une perte de vivacité.

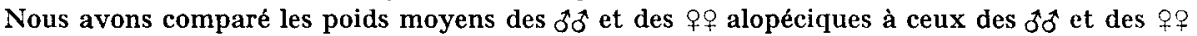

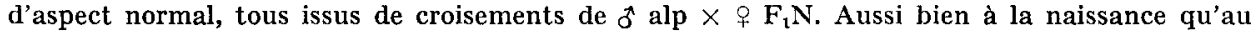
début de l'épilation ( $15^{\mathrm{e}}$ jour), il n'y a aucune différence pondérale significative entre les sujets qu'ils soient $\delta \widehat{\delta}$ ou 우으, alopéciques ou normaux. Au $21^{\mathrm{e}}$ jour (milieu de l'épilation) commence à se dessiner une différence de poids entre les $\delta^{*} \sigma^{\star}$ alopéciques et les $\delta^{*} \sigma^{*}$ normaux, en faveur de ces derniers; tandis que chez les 우우 cette différence n'apparait qu'au $30^{\mathrm{e}}$ jour, également en faveur des $ㅇ ㅜ$ normales. Les $ㅇ ㅜ$ alopéciques rattrapent le poids des sujets normaux vers le $60^{\circ}$ jour, tandis que pour les $\delta^{*} 0^{*}$ ce n'est que vers le $90^{\circ}$. Notons cependant que les $\delta^{*} \delta^{*}$ pèsent toujours plus lourd que les $+q$, qu'il s'agisse de sujets alopéciques ou normaux.

On sait le rôle que peut jouer, dans certains cas, l'action des glandes endocrines, tant sur la chute des poils que sur l'évolution du poids d'un sujet : une étude histologique est envisagée.

QUELQUES PRÉCISIONS SUR LE DÉTERMINISME HÉRÉDITAIRE DE LA FOURBURE EN RACE JERSEY D'AFRIQUE DU SUD

\author{
J.-G. Boyazoglu et E. MFyer. - Animal Husbandry and Dairy Research Institute, \\ Irene, Pretoria, République Sud Africaine \\ H.-P.-A. DE BOoM. - Faculty of Veterinary Science, Onderstepoort, Pretoria.
}

Depuis la fin des années 50, on a remarqué une anomalie des membres dans plusieurs troupeaux Jersey de la région du Cap. Les animaux paraissent normaux à la naissance mais, vers l'âge de 1 à 3 mois, leur démarche devient de plus en plus pénible sans qu'il soit possible d'y remédier par un traitement approprié. Une étude anatomique sur plus de vingt sujets disséqués à différents âges a montré qu'il s'agissait d'une fourbure; une étude toxicologique a permis d'écarter l'hypothèse d'une étiologie carentielle (de Boom et al., 1968, Proc. III Cong. S.A.G.S. July 1966, 58-62). L'étude génétique a débuté à Irene, en 1963. Un taureau anormal (A $b \mathrm{I}$ ) a été croisé à une dizaine de ses propres filles et petites-filles toutes phénotypiquement anormales. Des treize veaux nés, sept ont été suivis au-delà de 4 mois d'âge et tous étaient anormaux. $\mathrm{A} b_{\mathbf{t}}$ et un de ses fils anormal $\left(\mathrm{A} b_{2}\right)$ ont été ensuite croisés à 29 vaches normales non apparentées à eux. Les 33 veaux nés étaient en apparence normaux à l'âge adulte. Onze des génisses nées 